\title{
Quantization analysis of speckle intensity measurements for phase retrieval
}

\author{
Maallo, Anne Margarette S.; Almoro, Percival F.; Hanson, Steen Grüner
}

Published in:

Optical Society of America. Journal B: Optical Physics

Link to article, DOI:

10.1364/AO.49.005087

Publication date:

2010

Document Version

Publisher's PDF, also known as Version of record

Link back to DTU Orbit

Citation $(A P A)$ :

Maallo, A. M. S., Almoro, P. F., \& Hanson, S. G. (2010). Quantization analysis of speckle intensity

measurements for phase retrieval. Optical Society of America. Journal B: Optical Physics, 49(27), 5087-5094. https://doi.org/10.1364/AO.49.005087

\section{General rights}

Copyright and moral rights for the publications made accessible in the public portal are retained by the authors and/or other copyright owners and it is a condition of accessing publications that users recognise and abide by the legal requirements associated with these rights.

- Users may download and print one copy of any publication from the public portal for the purpose of private study or research.

- You may not further distribute the material or use it for any profit-making activity or commercial gain

- You may freely distribute the URL identifying the publication in the public portal 


\title{
Quantization analysis of speckle intensity measurements for phase retrieval
}

\author{
Anne Margarette S. Maallo, ${ }^{1, \star}$ Percival F. Almoro, ${ }^{1}$ and Steen G. Hanson ${ }^{2}$ \\ ${ }^{1}$ National Institute of Physics, University of the Philippines, Diliman, Quezon City 1101, Philippines \\ ${ }^{2}$ DTU-Fotonik, Department of Photonics Engineering, Danish Technical University, Roskilde 4000, Denmark \\ ${ }^{*}$ Corresponding author: marge.maallo@gmail.com
}

Received 10 July 2009; revised 14 August 2010; accepted 16 August 2010; posted 17 August 2010 (Doc. ID 114076); published 14 September 2010

\begin{abstract}
Speckle intensity measurements utilized for phase retrieval (PR) are sequentially taken with a digital camera, which introduces quantization error that diminishes the signal quality. Influences of quantization on the speckle intensity distribution and $P R$ are investigated numerically and experimentally in the static wavefront sensing setup. Results show that 3 to 4 bits are adequate to represent the speckle intensities and yield acceptable reconstructions at relatively fast convergence rates. Computer memory requirements may be eased down by 2.4 times if a 4 bit instead of an 8 bit camera is used. This may facilitate rapid speckle data acquisition for dynamic wavefront sensing. () 2010 Optical Society of America
\end{abstract}

OCIS codes: $\quad 110.2970,100.5070,100.3010,030.6140,010.7350,280.4788$.

\section{Introduction}

Quantization is a nonlinear analog-to-digital conversion process that generally results in information compression [1]. For applications that require highly accurate digital representation of a physical signal, the effects of this process are critical. The level of quantization depends on the bit depth of the measuring device $[2,3]$. Convenient digital cameras usually have 16 or 8 bits. To reduce quantization effects, high-bit-depth devices that tend to be quite expensive are used. Images recorded using these devices, however, require large memory allocations. In most applications, processing of large files is not optimal to a system's performance.

Like in conventional imaging, the effects of quantization error $(\mathrm{QE})$ in phase retrieval $(\mathrm{PR})$ arrive in the recording of the intensity patterns. In conventional iterative PR methods that utilize a single Fourier intensity measurement and a priori knowledge at the pupil plane, the effects of $\mathrm{QE}$ are pronounced [3]. The strong Fourier intensity peak dominates the more important high-frequency com-

0003-6935/10/275087-08\$15.00/0

(C) 2010 Optical Society of America ponents, which, in turn, are manifested as relatively weaker signals. Using a camera at a given bit depth having a finite number of gray levels, such weak signals will not be adequately represented. This condition may lead to slow convergence or nonconvergence in iterative reconstruction algorithms. In addition, the a priori knowledge of object area of support and nonnegativity constraints limits the choice of test objects for these methods. Recently, iterative PR methods that utilize multiple Fresnel intensities have been demonstrated. The multiple-intensity approach has been carried out through modulations of the aperture plane [4], light source wavelength [5], or detector position [6] . The use of multiple-intensity measurements, in principle, enhances the signal-to-noise ratio (SNR), thus, minimizing the effects of $\mathrm{QE}$.

In the PR that uses a volume speckle field (VSF) [6], the speckle intensity patterns are captured at 20 axially displaced positions. It is remarked that, for this technique, no a priori knowledge of the pupil plane is required; hence, it is not restrictive to the choice of test objects. Compared to holographic or interferometric methods, the advantage of the $\mathrm{PR}$ technique is the simple setup since no reference beam is required. In addition, reference-beam-induced aberrations in holographic/interferometric methods are avoided 
[7]. Compared to PR methods that use a single Fourier intensity [3], the multiple measurements in the speckle-based PR technique efficiently represent the important high-frequency components [7] corresponding to the fine details of a test object. In addition, a significant speckle intensity variation between the axial positions leads to a nonstagnating phase reconstruction. The technique has been utilized in aberration analysis [7] and in metrological applications [8] through static wavefront sensing. In a proposed design for parallel capture of speckle patterns for dynamic wavefront sensing [9], the simultaneous speckle data recording in a continuous operation requires large computer memory allocation.

Recently, an experimental demonstration of rapid speckle recording at a single plane using a spatial light modulator (SLM) was reported [10]. The SLM at a Fourier plane displays varying transfer functions representing free-space propagations over varying distances. The resulting speckle intensity patterns are then captured sequentially at a fixed detector plane [10]. The acquisition rate at which the multiple speckle images are captured and stored in the computer depends on the refresh rate of the SLM. It is remarked that the data transmission in the serial architecture of the computer data buses also affects the data capture rate. Thus, the speckle data file size should also be optimized to achieve faster transmission rates. Comparing data file sizes, the speckle patterns captured with an 8 bit camera is of smaller file size than those captured with a 16 bit camera. It is also remarked that another way to enhance the data transmission rate is by reducing the exposure time of the detector. Low exposure of the camera could mean using an effectively lower bitdepth detector from 8 bits to, for example, 4 bits. If this exposure setting is still sufficient, the exposure time can be reduced to a considerable factor of 16 , thus speeding up the whole system. In this case, however, the effects of camera noise (dark current) should be considered. Fast data acquisition will facilitate near-real-time wavefront reconstruction and, possibly, for remote processing over the Internet. Data acquisition is now often done in one station or laboratory and then transmitted to another, between collaborating researchers. Therefore, optimizations of the camera bit depth and memory allocation are imperative for the optimum utilization of any multiple-intensity reconstruction methods. Fields benefitting from real-time data acquisition and interpretation include, but are not limited to, biomedicine [11], and pharmaceutical science [12].

In this study, we investigate the influences of the camera bit depth on the quality of reconstructions and rate of convergence using the iterative specklebased PR technique. Quantization principles are presented in Section 2 and the principles of the PR technique are described in Section $\underline{3}$. Section $\underline{4}$ describes the parameters used in the simulations and experiments on the influences of the camera bit depth. Results and discussions are presented in
Section 5. Finally, in the last section, the summary, conclusions, and outlook are presented.

\section{Principles of Quantization}

Quantization has been studied as a tool for data compression $[2,13]$. In the field of coherent metrology, the effects of quantization in phase-shifting interferometry $[14,15]$, fringe analysis [1], and phase-shifting digital holography (PSDH) $[\underline{1} \overline{6}, 17]$ have been studied. It is remarked that, for the two former techniques, the phase information is derived only at the imaging plane. For PSDH, as well as for the speckle-based PR technique, the complex wave is reconstructed at different planes $[\underline{6}-\underline{10}, \underline{18}, \underline{19}]$; hence, a deeper study is warranted.

According to Skydan et al., quantization is a nonlinear process that involves rounding or truncating values of a continuous signal into discrete levels by a digital measurement device [1]. Figure 1 shows the quantization of a continuous signal into discrete values. The signal is incident on a digital device that introduces the quantization process. The signal is then cast into discrete digital levels $I_{Q}\left(x^{\prime}, y^{\prime}\right)$ given by Eq. (1) [16]:

$$
I_{Q}\left(x^{\prime}, y^{\prime}\right)=\operatorname{int}\left[\frac{I(x, y) B}{I_{\max }}\right] \times \frac{I_{\max }}{B} .
$$

In Eq. (1), $I_{\max }$ is the maximum value of the original signal $\bar{I}(x, y)$ and int is the digitizing function. Parts of the signal are grouped together and represented by a single value. $B$, which is the maximum discrete value given device with bit depth $N$ is [16]

$$
B=2^{N}-1 .
$$

In imaging applications, after quantization, the signal is represented as an image $I_{S}$ with a finite number of gray levels and is subsequently stored digitally. The lower the bit depth of the measuring device, the fewer gray levels are available. In case of 8 bits, the image is stored with integral pixel values given by Eq. (3):

$$
I_{S}(i, j)=\operatorname{int}\left[\frac{I_{Q}\left(x^{\prime}, y^{\prime}\right)}{\max \left(I_{Q}\right)} \times 255\right]
$$

In speckle-based PR, the ubiquitous speckle grains, which carry the high-frequency components, have a high dynamic range (difference between maximum

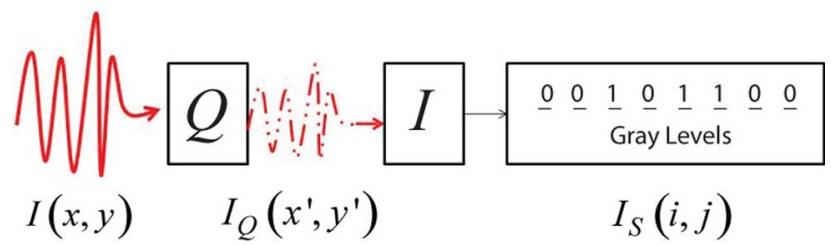

Fig. 1. (Color online) Quantization process. A continuous signal $(I(x, y))$ is cast into discrete values $\left(I_{Q}\left(x^{\prime}, y^{\prime}\right)\right)$ when passed through a digitizing device $(Q)$. This is then saved as an image $\left(I_{s}(i, j)\right)$ in computer $I$ with finite gray levels based on the bit depth of $Q$. 
and minimum detectable values). The favorable high dynamic range of the recorded speckle pattern and the averaging effects on the $\mathrm{QE}$ afforded by the multiple recordings increase the SNR. For signals with a high dynamic range, lowering of the bit depth may result in skewed images [16]. However, overrating may require unnecessary larger memory allocations. Reducing the bit depth leads to smaller data sizes and possibly faster data acquisition through easier data transmission. This feature is important for near-real-time processing of dynamic wavefronts in mobile or on-site data gathering or processing from a remote computer. It was shown by Mills and Yamaguchi that, in PSDH, at least 4 bits are needed to attain quality image reconstruction [16]. For PR using the hybrid input-output algorithm, a multispectral method for correcting the quantization errors was proposed by Yang and Takajo [3].

\section{Speckle Recording and Reconstruction Algorithm}

PR techniques that use a VSF may be categorized according to the distribution of the test object wavefront and the necessity of using a diffusing element. For the case of rough objects, the test wavefront is a VSF $[10,18,19]$, hence, the setup construction does not necessitate the use of a diffuser. For the case of a smooth object, such as a lens or a transparent biological specimen, the wavefront phase has a smooth curvature and the wavefront intensity does not vary significantly in the axial direction. Thus, for the case of a smooth object, the introduction of the phase diffuser facilitates the formation of the VSF. For the setup with a diffuser, the technique may be subclassified depending on the relative position of the diffuser and test object. A test object wavefront may illuminate the phase diffuser [8] or a speckle field that is formed when a plane wave is incident on the diffuser may be used to illuminate the test object [7]. In both cases, the purpose of the diffuser is to create a VSF. The diffuser converts a low-curvature wavefront into a rapidly varying detectable signal. A detector then samples the VSF sequentially, beginning at a distance $z_{0}$ from the diffuser aperture plane given by Eq. ()ㅜ [20]:

$$
z_{0}=\frac{\Lambda_{\text {trans }} D}{\lambda} .
$$

In this equation, $\Lambda_{\text {trans }}$ is the mean transverse speckle size at that measurement plane and $D$ is the diameter of the spot illuminated by the source with a given $\lambda$. To satisfy the sampling criterion, it is required that $\Lambda_{\text {trans }}$ is at least twice the detector pixel size $\Delta x$ at plane $z_{0}$ :

$$
\Lambda_{\text {trans }}=2 \Delta x .
$$

Constant increments $\Delta z$ in the recording distance are added until 20 images are taken. The value for $\Delta z$ used in the experiments is kept within the mean longitudinal speckle size:

$$
\Delta z=\Lambda_{\text {long }}=8 \lambda\left(\frac{z_{o}}{D}\right)^{2} .
$$

According to a Gerchberg-Saxton principle, phase reconstruction through an iterative PR algorithm depends not on the intensity measurements, per se, but on the variation between the intensity measurements [21]. This principle implies that a sufficient intensity variation is crucial in an iterative PR method to avoid the stagnation problem, where the phase calculation converges to a local minimum value. In the speckle-based PR technique, the diffuser used has physical characteristics that facilitate the partial scattering and partial transmission of the incident wave, or collectively, the formation of a partially developed speckle field (PDSF) [6]. The indentation on the diffuser surface introduces a maximum phase shift of less than $\pi$ on the incident field [6]. This condition is achieved when the maximum roughness height of the diffuser is less than the $\lambda$ of the incident light, and the illuminated spot size is much greater than the lateral scale on the surface [6]. During the speckle recording at axially displaced planes, the overlap of the unperturbed wave and the scattered wave results in a significant axial intensity variation, which is beneficial for a nonstagnating iterative PR algorithm.

The importance of using a PDSF has been demonstrated experimentally by comparisons with a setup without any diffuser [22] and, in another study [23], with a setup using an ordinary ground glass diffuser. The setup without any diffuser means the smooth test object is illuminated with a plane wave. The setup with a ground glass diffuser means the smooth test object is illuminated by a fully developed speckle field. It was shown that, without any diffuser in the setup, the diffracted light from the smooth test object does not exhibit significant intensity variation in the axially displaced measurement planes, thus, no reconstructions are obtained [22]. In [23], an experimental demonstration shows that the illumination of a smooth object with a fully developed speckle field also does not yield any reconstructions. It is emphasized that, when a fully developed speckle field is used in the illumination of the smooth test object, the resulting purely scattered wave does not exhibit sufficient axial intensity variation compared to the case when the speckle field is partially developed.

In the reconstruction algorithm, the wavefront calculation is based on the angular spectrum method of solving the Rayleigh-Sommerfeld (RS) wave propagation equation [6]. The wave amplitudes $(A)$ at each of the sampling planes are derived from the square roots of the sampled intensity patterns $\left(I_{S}\right)$ :

$$
A(i, j)=\sqrt{I_{S}(i, j)} .
$$

These are used as the constraints in the reconstruction algorithm. Unavoidably, the QE is propagated in 
the reconstruction process and affects the quality of the reconstructed phases. Initially, a random phase is used together with the measured amplitude in the first plane to form an estimate of the complex wave. This complex wave is then propagated to the second plane using the RS equation. The measured amplitude at the second plane is combined with the phase extracted from the wave propagation, and the resulting field is then propagated to the third plane. The forward direction of propagation is repeated until the wave from the 19th plane is propagated to the 20th plane (last plane). The wave from the last plane is sent back to the 19th plane, beginning the backward propagation [9]. Iterations are done to refine the phase reconstruction and are stopped if the retrieved phase from the last one varies by a small amount. A faster rate of convergence with an increasing number of intensity measurements and iterations has been previously reported [9]. For display purposes, the reconstructed phase is numerically filtered to remove the rapidly varying phase of the scattered wave component of the PDSF to obtain the desired test wavefront. Further discussions on the principles, reconstruction equation and other experimental techniques of the speckle-based PR method may be found in $[\underline{6}-10,18,22,24,25]$.

\section{Numerical and Experimental Parameters for Bit- Depth Lowering}

A detector area is simulated to have $1024 \times 1024$ pixels with pixel dimensions of $5.2 \mu \mathrm{m} \times 5.2 \mu \mathrm{m}$. To avoid numerical aliasing, a circular aperture is simulated to be placed in front of the test wavefront with a radius of 100 pixels. The test wavefront has a smooth spherical phase and uniform amplitude, as shown in Fig. 2(a). A diffuser plate is simulated to have a roughness height of $\lambda / 5$ with a refractive index of 1.85. A set of 20 intensity speckle images is taken beginning at $z_{0}=10 \mathrm{~mm}$ with $\Delta z=1 \mathrm{~mm}$. At each simulation plane, the VSF is imaged with all the different bit depths ( $N=1$ to 8$)$ according to Eqs. (1) and (2) before proceeding to the next measurement plane. This ensures a constant input signal for all the bit depths. In the characterizations of the intensity measurements and the reconstructions, normalized correlations of two one-dimensional (two-dimensional) functions are carried out using the built-in function corr (corr2) in MATLAB R2007. The correlation values for the different bit depths are normalized using the value of the autocorrelation for $N=8$. The corr function computes the Pearson's linear correlation coefficient between each pair of columns in the two functions. The corr 2 computes the correlation coefficient between two matrices.

In the experiments, a speckle field from the diffuser is used to illuminate the test object, which is a positive phase resolution target. During its fabrication, the details of a target exposed to ultraviolet light become soluble to the photoresist developer. The thickness of the photoresist (refractive index, 1.65) as measured using a Dektak profilometer is
$1.95 \mu \mathrm{m}$. The transmitted VSF is imaged at multiple planes with an 8 bit camera (CMOS, Opticstar, $1280(\mathrm{H}) \times 1024(\mathrm{~V}), 5.2 \mu \mathrm{m} \times 5.2 \mu \mathrm{m}$ pixel size $)$. The speckle images are numerically processed to reduce the bit depth according to Eq. (1).

\section{Results and Discussion}

Figures $2(\mathrm{~b})-2(\mathrm{f})$ are sample speckle images corresponding to $N=8,4,3,2$, and 1 , respectively, that show the effects of quantization on the image quality. The normalized pixel density histogram in Fig. 2(g) is evidence of the lower bit depths corresponding to lesser gray levels. Only the data from 8, 4, and 2 bits \are presented for simplicity. At 8 bits, almost all of the gray levels have a nonzero pixel density. This means that the information is widely spread over all the 256 levels ranging from 0 (black) to 255 (white). On the other hand, at 2 bits, only two levels are

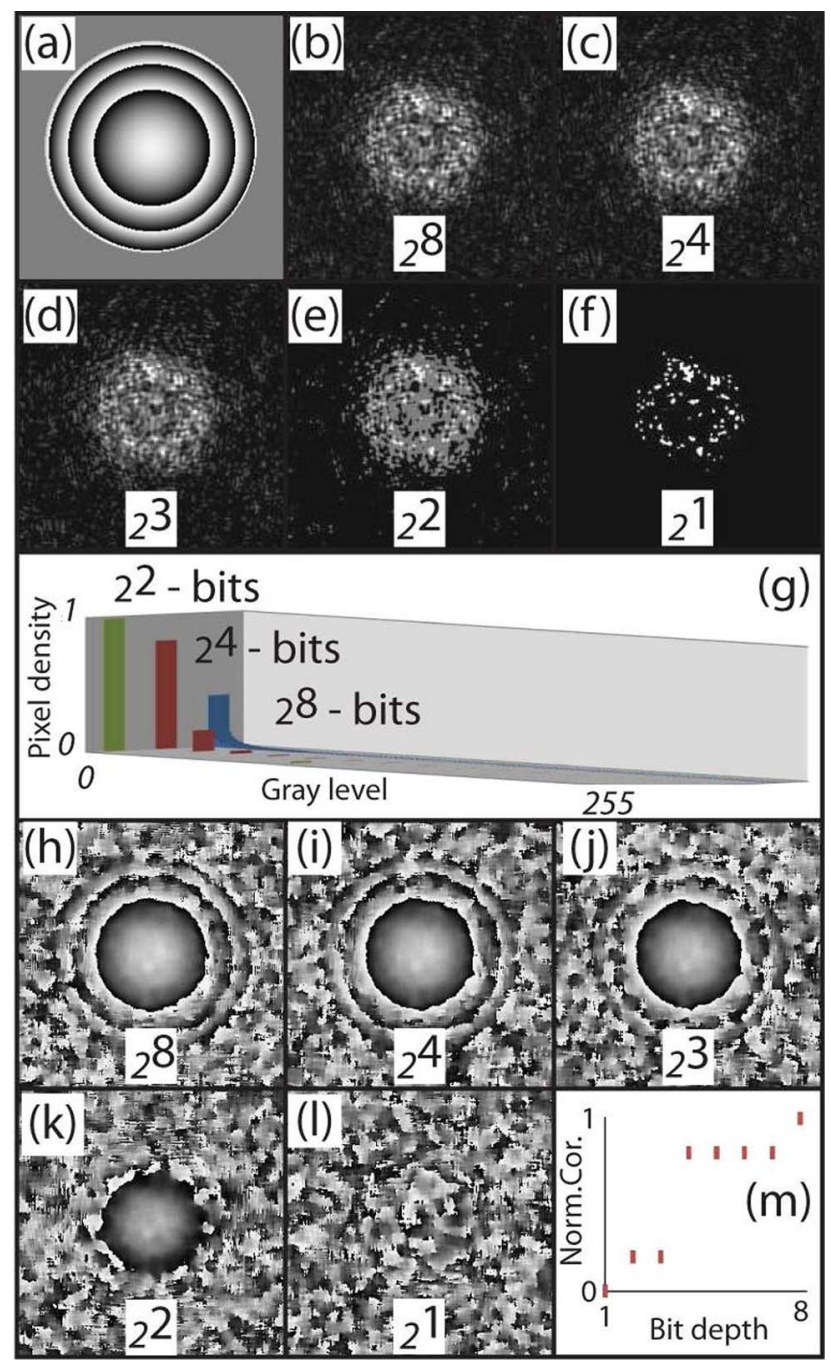

Fig. 2. (Color online) Numerical simulations of speckle patterns and reconstructions for different bit depths. (a) is the input smooth test wavefront. (b)-(f) are sampled speckle images at different bit depths. (g) is the pixel density histogram for bit depths $N=2,4$, and 8. (h)-(l) are phase reconstructions for different bit depths, and $(\mathrm{m})$ is the normalized correlation plot, with the reconstruction from $N=8$ as the standard. 
easily distinguishable, since the plot shows a normalized distribution. The population occupying the two higher gray levels is negligible compared to those occupying 0 . The number of occupied gray levels is critical in intensity-based phase reconstruction methods [3]. Figures 2(h)-2(l) show sample phase reconstructions after $i=10$ iterations corresponding to speckle patterns sampled at bit depths $N=8,4,3,2$, and 1, respectively. Qualitatively, wavefronts for $N>$ 1 show phase reconstructions, especially in the central region of the wavefront. It is also noted that a 3 bit digital camera may be considered sufficient in the imaging of the speckle patterns and eventually in reconstructing the particular test wavefront. However, based on the plot of normalized correlation of the reconstructions versus the bit depth using 8 bits as the standard [Fig. 2(m)], at least 4 bits are necessary to attain good reconstructions. It is noted that the normalized correlations of the reconstructions at $N=3,4,5,6$, and 7 are comparable. The dip in the correlation from $N=8$ to $N=7$ down to 4 is attributed to the unavoidable information loss due to the reduction in the number of gray levels.

To further understand the correlation trend, axial and transverse intensity line scans of the VSF are investigated. The ideal axial intensity distribution for a nonstagnating reconstruction algorithm is contingent on significant intensity variations between planes. Figure 3(a) shows the diagram for the axial line scan where the values for the same image pixel from all the speckle patterns are plotted. It is assumed this pixel is representative of the intensity behavior of the whole image for each particular plane - that is, the pixel values throughout an image will change accordingly in each plane. Figure 3(b) shows the intensity scans taken over the 20 measurement planes for 8, 4, 3, 2, and 1 bit depths. The line scan at 4 bits shows similar axial intensity variation as the line scan at 8 bits. For the line scans at 3 and 2 bits, only nominal intensity variations are seen. This decrease in intensity variation is even more evident in the line scan at 1 bit, where the variation is already considered negligible, changing only after the first plane and after the 19th plane. Figure 3(c) shows the correlation of the bit-lowered axial line scans to the 8 bit line scan. It is emphasized that correlations reveal the similarities of the line scans and, in effect, of the speckle images at different bit depths. The line scan at 4 bits is highly correlated with the lines scan for 5 to 8 bits. It is, therefore, safe to assume that this finding supports the result of the correlation of the phase reconstructions presented in Fig. 2. This also indicates that the speckle images sampled at 4 to 8 bit depths have almost the same value in terms of storing the information needed for successful PR.

Figure 3(d) depicts the transverse intensity line scan. A set of 20 adjacent pixels is chosen on the first image for each bit-depth value and a transverse line scan is made through this set. The ideal transverse speckle intensity scan should exhibit a high dynamic range and well-distributed gray level values. It is assumed that this set is representative for the behavior of all the pixels across images of the VSF. Other sets of 20 pixels from the same plane are assumed to vary in a similar manner as the one presented in Fig. 3(e). For all bit depths, the difference between the maximum and minimum intensity gray levels is the same, indicating a high dynamic range. It is noted, however, that only line scans greater than 3 bits show similar intensity distribution as the line scan for 8 bits. Figure $3(\mathrm{f})$ shows the correlation of the bit-lowered transverse line scans to the 8 bit line scan. The correlation plot shows the same trend as the axial line scan. This further supports the claim that recording the speckle images at 4 bits is sufficient for PR. The axial and transverse correlation plots also show that devices with bit depths lower than 3 bits do not have enough gray levels for depicting the speckle intensity variation from plane to plane and across a measurement plane. This may lead to stagnation in the algorithm as evident in the nonreconstructions from speckle images with bit depths of 2 and 1, as shown in Figs. 2(k) and 2(l), respectively. This indicates that the optimum bit depth is 4 bits, which provides a good trade-off between memory requirement and reconstruction efficiency. It is remarked that, for comparisons between reconstructions from speckle patterns sampled using higher bit-depth devices $(N>8$ bits), this optimum value will not change.

The main benefit of using a device with a low bit depth is the reduction of memory requirements. The memory used by each image at different bit depths is summed for the whole VSF sample. Figure 4 shows a

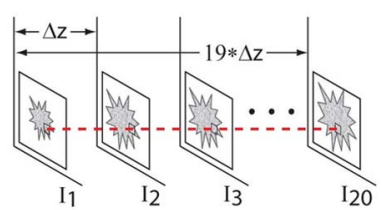

(a) Axial scan diagram

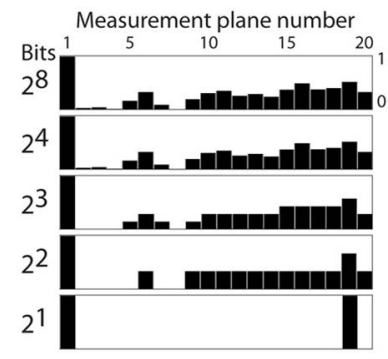

(b) Axial intens. scan

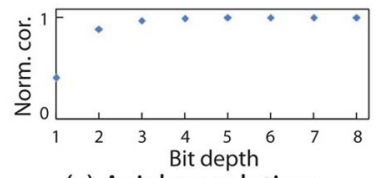

(c) Axial correlations

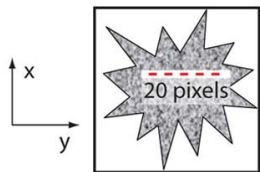

(d) Transverse scan diagram

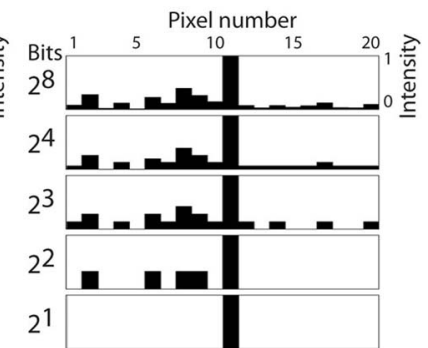

(e) Transverse intens. scan

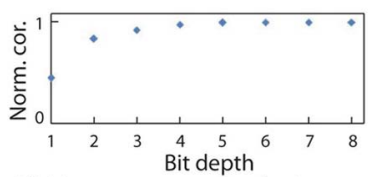

(f) Transverse correlations
Fig. 3. (Color online) (a)-(c) Axial and (d)-(f) transverse intensity scans of a VSF and the corresponding correlations for different bit depths. Axial and transverse line scans obtained at bit depth $N=$ 8 are used as standards. 
plot of the memory allocation for all 20 images of the VSF at an array size of $1024 \times 1024$. Using the maximum bit depth of the typical digital camera takes up 7.13 MB of storage space for the speckle patterns. If a device with just 4 bits is used, all the images use only $2.95 \mathrm{MB}$ of memory. This decreases the storage requirement by up to 2.4 times. It is remarked that smaller memory requirements afforded by low bit-depth cameras do not lead to faster algorithm processing time. It was found that the more relevant parameter for faster processing is the speckle array size. The influences of array size on the computational speed and resolution are beyond the scope of the present study.

Figure 5 shows the experimental results. The effects of bit depth on the intensity variation are manifested on the performance of the PR algorithm in reconstructing the object wavefront. The characteristics of the intensity distribution for different bit depths are investigated in the axial and transverse directions. For the transverse direction, Figs. 5(a)$5(\mathrm{~d})$ are the speckle patterns at the first measurement plane for bit depths of $1,2,3$, and 8, respectively. Also shown in Figs. 5(a)-5(d) are intensity plots taken over 20 pixels. The line scan at 3 bits [Fig. 5(c)] shows intensity variation similar to the line scan at 8 bits. For the axial direction, Figs. 5(e)-5(h) are the intensity plots of a specific image line (along the $y$ axis) scanned over the 20 measurement planes. The zoomed-in sections over six planes show that the line scan at 3 bits has variation similar to that of the line scan at 8 bits. Figures 5(i)-5(l) show the phase plots from the PR algorithm using sets of speckle patterns at bit depths of 1, 2, 3, and 8, respectively. Evidently, good reconstructions are observed for bit depths of 3 and higher.

The influence of bit depth on the convergence rate of the iterative processing is further investigated. The phase map obtained for $N=8$ and $i=10$ is used as standard. The difference between the phase map for a given $N$ and $i$ and the standard phase map is evaluated using root mean square deviation (RMSD). To quantify the rate of convergence for increasing numbers of iterations, a numerical value for the RMSD is set as a threshold value to indicate that the iterative phase calculation has converged. In this study, the threshold value is chosen based on quality features exhibited in the phase map.

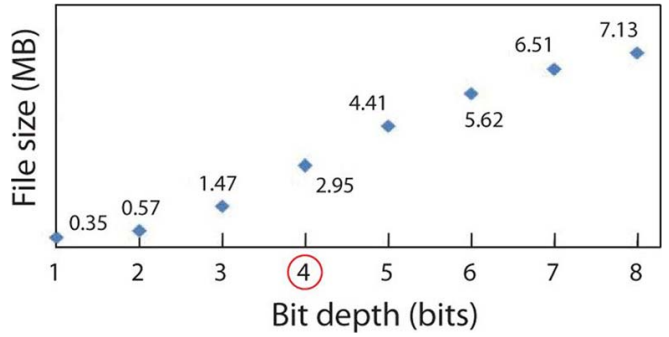

Fig. 4. (Color online) Memory requirements for 20 speckle patterns at different bit depths.
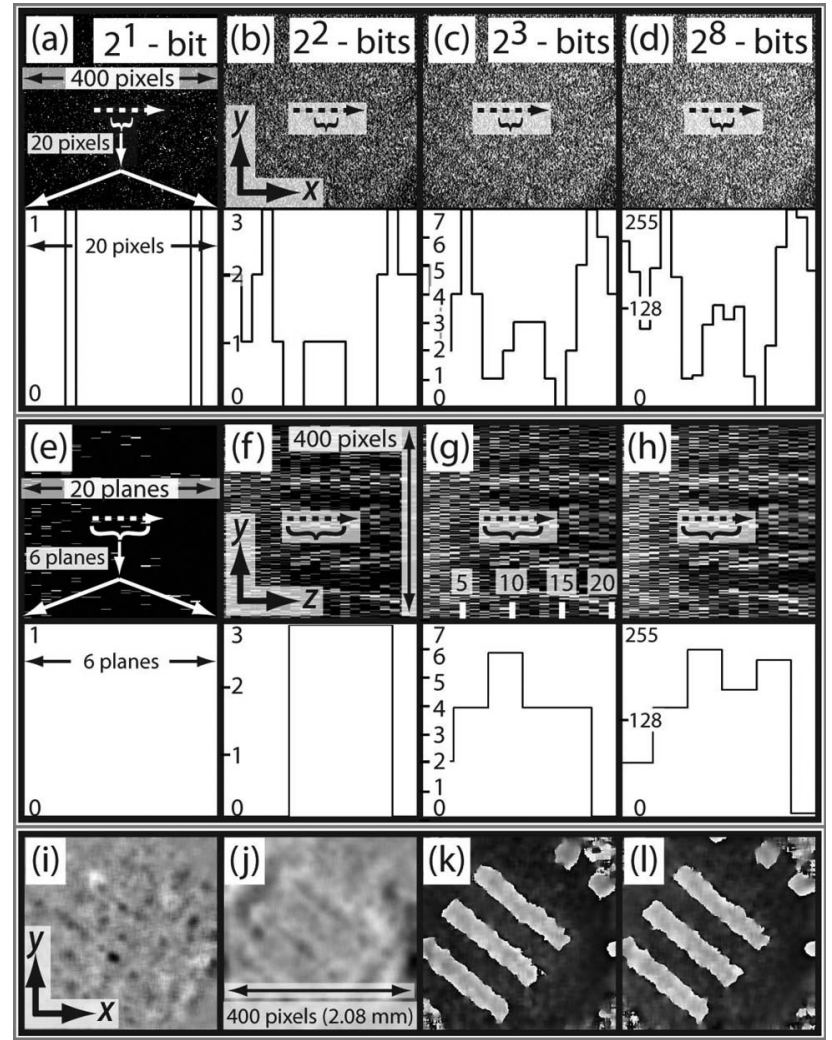

Fig. 5. Experimental results. (a)-(d) and (b)-(h) are the speckle intensity line scans, in the transverse and axial directions, respectively, for bit depths $N=1,2,3$, and 8 (left to right). (i)-(l) are the corresponding reconstructed phase maps.

Figures $6(\mathrm{a})$ and $6(\mathrm{~b})$ show representative phase difference maps for $\bar{N}=3$ and $N=4$, respectively. From left to right, the images are arranged in order
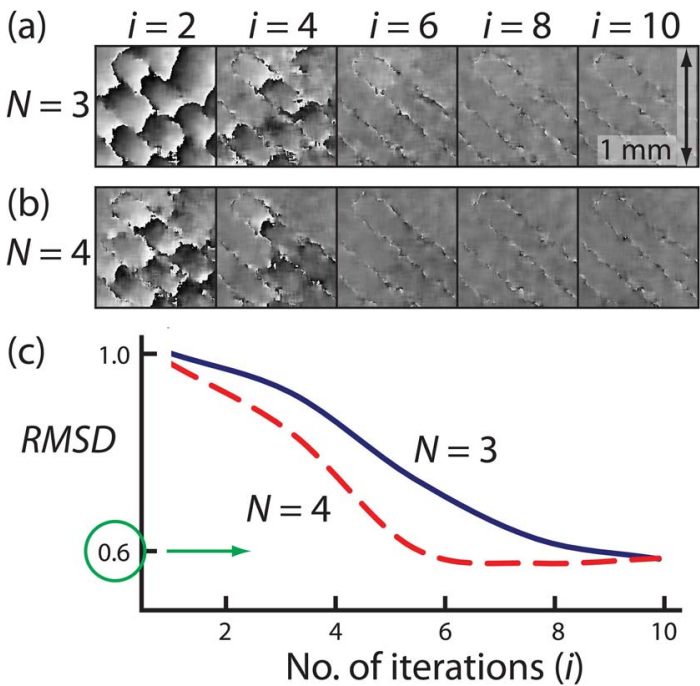

Fig. 6. (Color online) Influences of bit depth and number of iterations on the rate of convergence. (a) and (b) show portions of the phase difference maps for increasing numbers of iterations, $i$, (left to right) for bit depths $N=3$ and 4 , respectively. Phase map obtained for $N=8$ and $i=10$ [Fig. 5(1)] is used as standard. (c) Graphs depicting the rates of decrease, for $N=3$ and 4 , in the RMSD of the phase difference maps. 
of increasing number of iterations $(i=2,4, \ldots, 10)$. For $N=3$ [Fig. 6(a)], a significant reduction of residual phase structures is observed after eight iterations. For $N=4$ [Fig. 6(b)], this condition occurred after six iterations. It is remarked that no error reductions are observed for $N=2$ and $N=1$, for increasing iterations up to $i=1000$ (not shown). A composite plot of the RMSDs for $N=3$ and 4, for increasing iteration numbers, is shown in Fig. 6(c). Normalization is based on the RMSD for $N \overline{=3}$, $i=1$. The graphs show that the RMSDs decrease as the number of iterations increases, for both $N=$ 3 (solid curve) and 4 (dashed curve). Using a threshold value of 0.6 , the phase calculation for $N=3$ is considered converged after eight iterations. The rate of convergence for $N=4$ is slightly higher, since the reconstruction is considered converged after $i=6$.

\section{Summary, Conclusions, and Outlook}

The effects of quantization due to the finite bit depth of the digital sampling device are studied in relation to the basic principles of the speckle-based PR technique to address memory optimization. Simulations are done to probe phase reconstructions, along with experiments. From the simulations, it is shown that the phase can be reconstructed using a camera with bit depth as low as 3 bits. But to get a quality comparable to the reconstruction from images taken with 8 bits and from the same number of iterations, at least 4 bits is needed. This may lighten the memory requirement in actual implementation by 2.4 times. The results based on experimental data agree with the results from the numerical study. The correlation values between the actual speckle images show that the speckle patterns recorded at 3 bits up to 8 bits are highly correlated. Thus, the phase reconstructions from $N=3$ and $N=8$ images are also highly correlated. The rate of convergence increases with bit depth. It can be said that a device with $N=3$ corresponding to eight discrete gray levels is sufficient in representing the intensity changes between and across the VSF planes.

Finally, a major development is seen in the direction of enhanced data acquisition rates using a SLM [10] and, possibly, other active optical devices. Toward this, an ongoing study analyzes a design for a single-plane multiple speckle pattern $P R$ technique using a deformable mirror [26] within the formalism of complex ABCD matrices, facilitating its use in conjunction with dynamic objects [27]. The variable focal length deformable mirror positioned at a Fourier plane of a lens comprises the adaptive optical system that replaces the time-consuming axial displacements in the conventional free-space multiple plane setup. Compared with a SLM, a deformable mirror has a smooth continuous surface that avoids pixelation, pixel cross talk, and nonplanarity issues. Another development is the use of an imaging system, which gives flexibility of magnification and demagnification of the test wavefronts. Experimental implementations using PR with an imaging setup will be reported in [23]. Future work is also geared toward applications of the technique in nondestructive testing of birefringent materials, optical vortices in photonic crystal fibers, and depth-of-field extension in phase microscopy.

P. Almoro and A. Maallo gratefully acknowledge the Office of the Chancellor, in collaboration with the Office of the Vice-Chancellor for Research and Development, of the University of the Philippines Diliman for funding support through the Outright Research Grant. A. Maallo also acknowledges the Department of Science and Technology-Philippine Council for Advanced Science and Technology Research and Development (ASTHRDP)for the scholarship grant. The authors acknowledge financial support from the Danish Council for Technology and Innovation under the Innovation Consortium Centre for Industrial Nano Optics (CINO).

\section{References}

1. O. Skydan, F. Lilley, M. Lalor, and D. Burton, "Quantization error of CCD cameras and their influence on phase calculations in fringe pattern analysis," Appl. Opt. 42, 5302-5307 (2003).

2. A. Shortt, T. Naughton, and B. Javidi, "A companding approach for nonuniform quantization of digital holograms of three dimensional objects," Opt. Express 14, 5129-5134 (2006).

3. S. Yang and H. Takajo, "Quantization error reduction in the measurement of Fourier intensity for phase retrieval," Jpn. J. Appl. Phys. 43, 5747-5751 (2004).

4. M. Guizar-Sicairos and J. R. Fienup, "Measurement of coherent x-ray focused beams by phase retrieval with transverse translation diversity," Opt. Express 17, 2670-2685 (2009).

5. P. Bao, F. Zhang, G. Pedrini, and W. Osten, "Phase retrieval using multiple illumination wavelengths," Opt. Lett. 33, 309-311 (2008)

6. P. F. Almoro and S. G. Hanson, "Random phase plate for wavefront sensing via phase retrieval and a volume speckle field," Appl. Opt. 47, 2979-2987 (2008).

7. P. F. Almoro, P. N. Gundu, and S. G. Hanson, "Numerical correction of aberrations via phase retrieval with speckle illumination," Opt. Lett. 34, 521-523 (2009).

8. P. F. Almoro, G. Pedrini, A. Anand, W. Osten, and S. G. Hanson, "Angular displacement and deformation analyses using a speckle-based wavefront sensor," Appl. Opt. 48, 932-940 (2009).

9. P. Almoro, A. Maallo, and S. Hanson, "Fast-convergent algorithm for speckle-based phase retrieval and a design for dynamic wavefront sensing," Appl. Opt. 48, 1485-1493 (2009).

10. C. Falldorf, M. Agour, C. v. Kopylow, and R. B. Bergmann, "Phase retrieval by means of a spatial light modulator in the Fourier domain of an imaging system," Appl. Opt. 49, 1826-1830 (2010).

11. M. Schirmer, M. Fujio, M. Minami, J. Miura, T. Araki, and T. Yasui, "Biomedical applications of a real-time terahertz color scanner," Biomed. Opt. Express 1, 354-366 (2010).

12. M. Možina, D. Tomaževiča, S. Lebenc, F. Pernuš, and B. Likara, "Digital imaging as a process analytical technology tool for fluid-bed pellet coating process," Euro. J. Pharm. Sci. 41, 156-162 (2010).

13. T. Naughton, Y. Frauel, B. Javidi, and E. Tajahuerce, "Compression of digital holograms for three-dimensional object reconstruction and recognition," Appl. Opt. 41, 41244132 (2002). 
14. B. Zhao and Y. Surrel, "Effect of quantization error on the computed phase of shifting measurements," Appl. Opt. 36, 2070-2075 (1997).

15. B. Zhao, "Effect of intensity-correlated error due to quantization and noise on phase-shifting method," Opt. Lasers Eng. 28, 199-211 (1997).

16. G. Mills and I. Yamaguchi, "Effects of quantization in phaseshifting digital holography," Appl. Opt. 44, 1216-1225 (2005).

17. I. Yamaguchi, K. Yamamoto, G. Mills, and M. Yakota, "Image reconstruction only by phase data in phase-shifting digital holography," Appl. Opt. 45, 975-983 (2006).

18. P. Almoro, G. Pedrini, and W. Osten, "Complete wavefront reconstruction using sequential intensity measurements of a volume speckle field," Appl. Opt. 45, 8596-8605 (2006).

19. G. Pedrini, W. Osten, and Y. Zhang, "Wave-front reconstruction from a sequence of interferograms recorded at different planes," Opt. Lett. 30, 833-835 (2005).

20. P. Jacquot, "Speckle interferometry: a review of the principal methods in use for experimental mechanics applications," Strain 44, 57-69 (2008).
21. R. W. Gerchberg and W. O. Saxton, "A practical algorithm for the determination of phase from image and diffraction plane pictures," Optik (Stuttg.) 35, 237-246 (1972).

22. P. F. Almoro and S. G. Hanson, "Object wave reconstruction by speckle illumination and phase retrieval,” J. Euro. Opt. Soc. Rapid Publ. 4, 09002 (2009).

23. P. F. Almoro, and S. G. Hanson are preparing a manuscript to be called "Enhanced wavefront reconstruction by random phase modulation with a phase diffuser."

24. P. F. Almoro, G. Pedrini, and W. Osten, "Aperture synthesis in phase retrieval using a volume-speckle field," Opt. Lett. 32, 733-735 (2007).

25. P. F. Almoro and S. G. Hanson, "Wavefront sensing using speckles with fringe compensation," Opt. Express 16, 76087618 (2008).

26. P. F. Almoro and S. G. Hanson are preparing a manuscript to be called "Single-plane multiple speckle pattern phase retrieval using a deformable mirror."

27. W. Wang, S. Hanson, and M. Takeda, "Complex amplitude correlations of dynamic laser speckle in complex ABCD optical systems," J. Opt. Soc. Am. A 23, 2198-2207 (2006). 